\title{
External Parameters Affecting on the Photoluminescence of InAs Spherical Layer Quantum Dot
}

\author{
Marwan Zuhair Elias \\ Biophysics Department, Sciences College, Mosul University, Mosul, Iraq \\ Email: marwanzt@uomosul.edu.iq
}

How to cite this paper: Elias, M.Z. (2021) External Parameters Affecting on the Photoluminescence of InAs Spherical Layer Quantum Dot. Journal of Applied Mathematics and Physics, 9, 2439-2446. https://doi.org/10.4236/jamp.2021.910155

Received: August 25, 2021

Accepted: October 11, 2021

Published: October 14, 2021

Copyright $\odot 2021$ by author(s) and Scientific Research Publishing Inc. This work is licensed under the Creative Commons Attribution International License (CC BY 4.0).

http://creativecommons.org/licenses/by/4.0/

\begin{abstract}
Spherical layer quantum dots (SLQDs) attract a great deal of importance, and have various optoelectronics applications due to their outstanding optical and electrical properties. The photoluminescence (PL) and the electroluminescence (EL) spectra of InAs (SLQDs) were investigated theoretically under the presence of external parameters (pressure, temperature, electric field). Existing of both the temperature and the applied electric field lead to a significant decrease in photoluminescence peak energy (red-shift), while an increase existed in presence of applied hydrostatic pressure (blue-shift). Also with increasing the quantum azimuthal number the photoluminescence peak energy increase. In addition, we found no effect on the band shape of the luminescence as a result of existing such parameters. The study indicates the importance of such parameters as fitting parameters for photoluminescence spectra.
\end{abstract}

\section{Keywords}

Layer Quantum Dots, Photoluminescence, Electroluminescence, Pressure-Temperature Dependence

\section{Introduction}

Studying the electronic devices using photoluminescence is a powerful technique to extract valuable information about semiconductor sample. The promising objects of quantum dots (QDs) are due to their wide range of applications, ranging from bio-labeling, photodetection, light emitting diode and solar cells. In the case of applications, numerous parameters may affect the photoluminescence (PL) of QDs [1]. The authors of [2] investigate the photoluminescence properties of core-shell InP/ZnS quantum dots under temperature from 80 - 300 
K with four different sizes by $405 \mathrm{~nm}$ continuous excitation light, the authors found that there was a red-shift in photoluminescence peak with increasing temperature which attributed to the interaction of longitudinal acoustic phonons with excitons. In Ref. [3], the authors studied the quantum dots photoluminescence of perovskite $\mathrm{CsPBr}_{3}$ enhanced by nanorods plasmonic Au. They found that the quantum dots film photoluminescence intensity of $\mathrm{Au}$ nanorods $/ \mathrm{Cs} \mathrm{PbBr}_{3}$ exhibits an enhancement of 2-fold compared with pristine $\mathrm{CsPbBr}_{3}$ quantum dots film. Kyun Geun Ung et al. [4] studied the perovskite quantum dots (PQDs) and reported an effective strategy for highly luminescent of (PQDs) by a simple doping. In Ref [5], the fabrication of hybrid nanoflowers graphene quantum dots (NFGQDs)/ZnO was investigated. The structure showed strong photoluminescence emissions were more efficient integration of NFGQDs exist as a result of the large $\mathrm{ZnO}$ surface area. Neslihan Ayarcı Kuruoğlu et al. [6] investigate the Carrier Conduction Mechanism over InAs/InP Quantum Dashes and InAs/GaAs Quantum Dots Based p-i-n Laser Heterostructures. The charge transfer characteristics of the long wavelength semiconductor laser structures, containing quantum dot layers (QDs), were investigated by means of temperature dependent current-voltage and electroluminescence measurements over InAs/InP, and InAs/GaAs based p-i-n structures, they found that the peak value of emitted laser light for InAs/InP QDashes and InAs/GaAs QDs occurred in $1.55 \mu \mathrm{m}$ and 1.3 $\mu \mathrm{m}$, respectively. The authors of [7] propose a synthesis method to synthesize $\mathrm{CsPbCl}_{3}: \mathrm{Mn}$ quantum dots using a single-step ultrasonic. The quantum dots synthesized improved the photoluminescence properties and exhibit both blue and orange Mn emissions. In Ref [8], the authors demonstrated the synthesis of scalable carbon quantum dots by simply heating or microwave using a mixture of ethylenediamine (EDA) and citric acid (CA) in presence of glycerol (Gly) as solvent of high boiling point. The CQDs showed UV absorption at about $346 \mathrm{~nm}$ and a broad spectrum of photoluminescence with quantum yield of $49.9 \%$.

Roberta De Angelis et al. [9] investigated the Chemical Sensitivity of Luminescent Epitaxial Surface InP Quantum Dots, where a broad-band near-infrared photoluminescence ranging from 750 to $865 \mathrm{~nm}$ was reported from the surface InP quantum dots grown by gas source molecular beam epitaxy on $\operatorname{In}_{0.48} \mathrm{Ga}_{0.52} \mathrm{P}$ buffer layer lattice matched to GaAs substrate. When the quantum dots exposed to vapors of different chemical solvents with the highest sensitivity for alcohol (methanol and ethanol) vapours a reversible luminescence intensity enhancement has been observed. The authors found that the luminescent behavior depends on the solvent type and concentration. In addition to that, they proved that the solvent vapor has no effect on the peak energy and band shape of the luminescence.

The authors of [10] studied the mechanisms of radiative recombination of large InAs/GaAs quantum dots, the large InAs/GaAs quantum dots optical properties were investigated by low-temperature photoluminescence versus excitation-power density. They concluded that to have more than one exciton by 
dots the probability must be considered, and we must revise the usual equation to correctly describe the origin of the recombination and must include other factors as relaxation time, radiative recombination rate, scattering, and others.

The investigation of layer quantum dots (SLQDs), in which motion of radial charge carriers limited on inner and outer radii are of great interest and allow flexible manipulations of the optical absorption of layer quantum structures [11].

In our present work, the effects of external parameters on the photoluminescence of (SLQDs) were investigated theoretically. We studied the ability of using electric field intensity, temperature and pressure, as fitting parameters on such characteristics.

\section{Theory}

\subsection{Pressure-Temperature Effects}

Consider the motion of electron in layer quantum dot of spherical shape (SLQDs) with inner radius $R_{1}$ and outer radius $R_{2}$. We consider the transition between both hole and electron states. According to Ref [11] and considering the effect of both temperature and pressure. The wave functions of (SLQD) are.

$$
\begin{aligned}
& \Psi_{n, l, m}^{e(h)}(r, \theta, \varphi, e, p, t) \\
& =\sqrt{\frac{\pi k(e, p, t)}{2 r}}\left[c_{1} \cdot J_{(l+1 / 2)}(k(e, p, t) \cdot r)+c_{2} \cdot J_{-(l+1 / 2)}(k(e, p, t) \cdot r)\right] y_{l, m}(\theta, \varphi)
\end{aligned}
$$

where $J_{ \pm(l+1 / 2)}$ are the Bessel functions, $I$ and $m$ are the azimuthal and the magnetic quantum numbers respectively, $C_{1(2)}$ are the constants of normalization. The wave function (1) should satisfy the boundary conditions (wave function vanishes outside the layer) the electron energy spectrum determined from the transcendent equation:

$$
\begin{aligned}
& J_{(l+1 / 2)}\left(k(e, p, t) \cdot R_{1}\right) \cdot J_{-(l+1 / 2)}\left(k(e, p, t) \cdot R_{2}\right) \\
& -J_{(l+1 / 2)}\left(k(e, p, t) \cdot R_{2}\right) \cdot J_{-(l+1 / 2)}\left(k(e, p, t) \cdot R_{1}\right)=0
\end{aligned}
$$

The absorption coefficient for the transition between the holes and the electron states of conduction band are:

$$
H\left(\omega, R_{1}, R_{2}, p, t\right)=A_{v} \sum_{\substack{l, l^{\prime} \\ m, m^{\prime}}}^{n, n^{\prime}}(2 l+1) \delta\left(\hbar \omega-E_{g}(p, t)-E_{n, l, m}^{e}-E_{n^{\prime}, l^{\prime}, m^{\prime}}^{h}\right)
$$

where $A_{v}$ coefficient proportional to the square of the modulus of the matrix elements, $n=1,2,3, m=0, \pm 1, \pm 2$ and $l=0,1,2, E_{g}(p, t)$ is the bulk InAs band gap given by:

$$
E_{g}(P, T)=\left(533+7.7 P-\frac{0.276 T^{2}}{T+83}\right) \mathrm{meV}
$$

$\omega$ is the frequency of the light, The selection rules according to Ref [12] will be,

$$
n^{e}=n^{h}, m^{e}=-m^{h}, l^{e}=l^{h}
$$

For the effective mass of the electron in presence of pressure-temperature effect will be [13]. 


$$
m^{*}(P, T)=\left[1+\frac{15020}{E_{g}(P, T)}+\frac{7510}{E_{g}(P, T)+341}\right]^{-1} m_{0},
$$

The fractional change in the volume of the spherical layer quantum dot is given by:

$$
R_{1(2)}(P)=R_{1(2)}(0)\left(1-\left(S_{11}+2 S_{12}\right) P\right)
$$

where $P$ is the pressure in (k.bar), $T$ is the temperature in $(\mathrm{K})$, and $m_{0}$ is the free electron mass. $S_{11}\left(=1.946 \times 10^{-3} \mathrm{kbar}^{-1}\right)$ and $S_{12}\left(=-6.855 \times 10^{-4} \mathrm{kbar}^{-1}\right)$ are the elastic constant of the InAs and $R_{1(2)}(0)$ is the inner (outer) zero-pressure radiuses.

\subsection{Presence of Electric Field}

Now let an electric field ( $V$ ) be applied uniformly on the system as perturbation. Then the corresponding energy in frames of perturbation theory has the form:

$$
E=\frac{p^{2}}{2 m}-e(\vec{V} \cdot \vec{r})
$$

Corrections of energy in presence of the electric field $V(r)$ were calculated using the following integrals:

$$
\left\langle l^{\prime}, m^{\prime}|\hat{V}| l, m\right\rangle=\int Y_{l^{\prime}, m^{\prime}}^{*}(\theta, \varphi) \hat{V}(\theta, \varphi) Y_{l, m}(\theta, \varphi) \mathrm{d} \Omega
$$

All diagonal elements equal zero [12], so the first-order correction of the perturbation theory will be zero:

$$
\Delta_{1} E_{l, m}=\langle l, m|\hat{V}| l, m\rangle=0 .
$$

For the second order correction and according to Ref [14] we can write:

$$
\begin{aligned}
\Delta E_{2}= & \sum_{n^{l}} \frac{I_{1}^{n^{\prime}, l+1} e^{2}}{E_{n, l}^{(0)}-E_{n, l+1}^{(0)}}\left(\frac{1}{4}\left|V_{x}-i V_{y}\right|^{2} a_{l, m}^{2}+\frac{1}{4}\left|V_{x}+i V_{y}\right|^{2} a_{l,-m}^{2}+\varepsilon_{z}^{2} b_{l, m}^{2}\right) \\
& +\sum_{n^{l}} \frac{I_{2}^{n^{\prime}, l-1} e^{2}}{E_{n, l}^{(0)}-E_{n, l-1}^{(0)}}\left(\frac{1}{4}\left|V_{x}-i V_{y}\right|^{2} a_{l-1, m-1}^{2}+\frac{1}{4}\left|V_{x}+i V_{y}\right|^{2} a_{l-1, m-1}^{2}+\varepsilon_{z}^{2} b_{l-1, m}^{2}\right)
\end{aligned}
$$

where $I_{1}^{n^{\prime}, l+1}, I_{1}^{n^{\prime}, l-1}, a_{l, m}$ and $b_{l, m}$ are according to [14].

The photoluminescence spectra for the cases under consideration are calculated using the relation [15]:

$$
P\left(\omega, R_{1}, R_{2}, p, t\right)=P_{0} \cdot \hbar \omega \cdot H\left(\omega, R_{1}, R_{2}, p, t\right) \cdot \mathrm{e}^{\frac{\hbar \omega-E_{g}}{K_{b} \cdot T}} \cdot \mathrm{e}^{\frac{0.5-E_{g}}{K_{b} \cdot T}}
$$

where $P_{0}$ is a coefficient proportional to the square of the modulus of the matrix elements

All calculations were made numerically by the finite element method using mathematica 5 .

\section{Results and Discussions}

Now, let us proceed to the results and discussions. Numerical calculations have been performed for the photoluminescence of (SLQDs). All needed parameters 
interring our numerical calculations were taken as a function of pressure and temperature for InAs. Let us consider the photoluminescence of (SLQDs) neglecting the interaction between the hole and electron within the framework of the regime of strong size quantization.

Figure 1 (left) represents the photoluminescence (in arbitrary units) versus the frequency of incident hole to electron transition $1 \mathrm{~h} \rightarrow 1 \mathrm{e}$ (in $\mathrm{meV}$ ) at different value of temperatures ( $T=100 \mathrm{~K}, 200 \mathrm{~K}, 300 \mathrm{~K}, 400 \mathrm{~K}$ ), fixed values of inner radius $\left(R_{1}=300 \AA\right)$, outer radius $\left(R_{2}=900 \AA\right)$, pressure $(P=10 \mathrm{k} \cdot \mathrm{bar})$ and different azimuthal numbers $\left(I_{c}=1,2,3\right)$. From the figures, there was a red-shift in photoluminescence peak energy and a decrease in intensity with increasing temperature which attributed to the interaction of excitons with longitudinal acoustic phonons [2]. In addition to that, we notice that the increase in temperature has no effect on the band shape of the luminescence [9]. Also we see the photoluminescence peak energy increase with increasing the quantum azimuthal number $l_{c}$ which may be attributed to the thermally escape of the carriers to higher energy levels [12] [16].

The opposite picture appears for photoluminescence peak energy Figure 1 (right) where there was a blue-shift in photoluminescence peak energy with increasing pressure at fixed value of temperature $(T=4 \mathrm{~K})$ which may be attributed to the energy dependencies on the applied pressure, where a change in the energy gap of InAs as a result of changing the applied pressure will exist lead to changing the Coulomb interaction energy as a result of an internal huge strain of the dot of InAs, which will change the electrons and holes quantization energies [12]. In addition to that Ref [13] showed that the interband emission energy increases when the hydrostatic pressure increases.

Figure 2 represents the photoluminescence (in arbitrary units) versus the
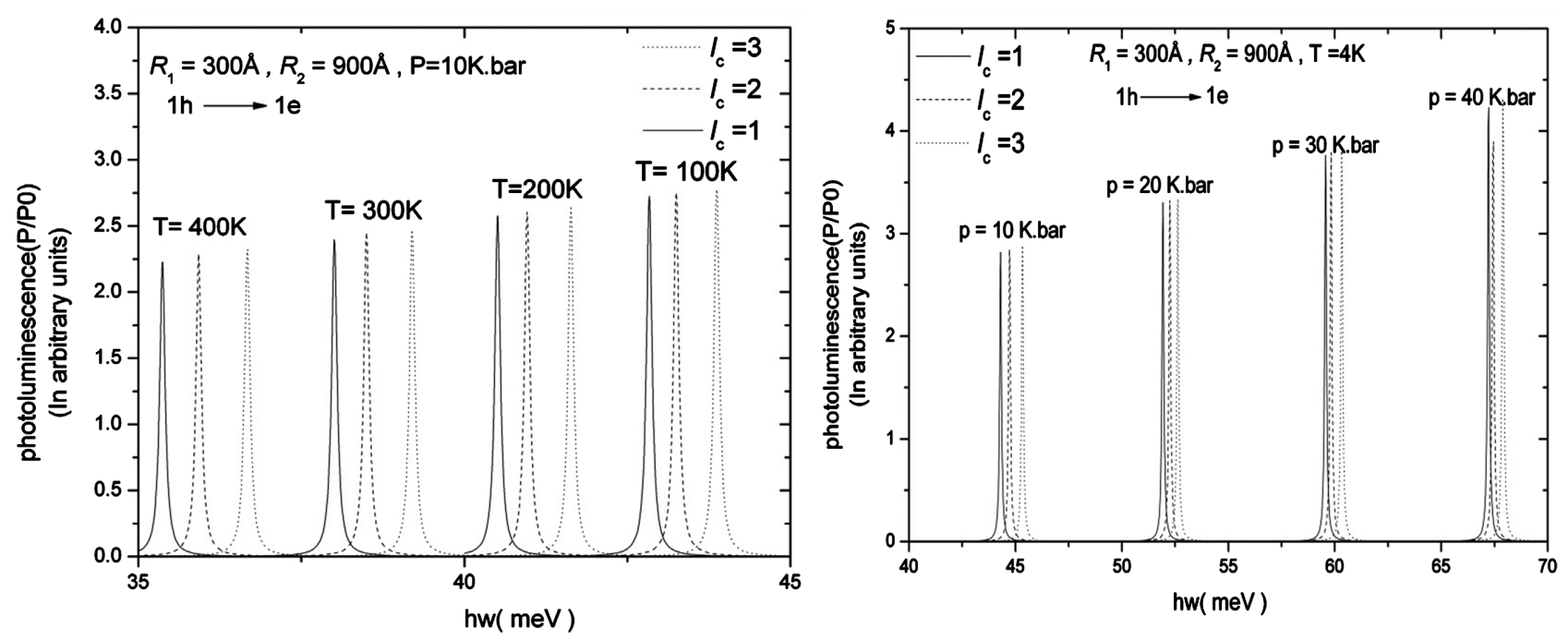

Figure 1. Photoluminescence (in arbitrary units) of InAs (SLQDs) versus the frequency of incident hole to electron transition $1 \mathrm{~h}$ $\rightarrow 1 \mathrm{e}$ (in $\mathrm{meV})$ at fixed values of inner and outer radiuses $\left(R_{1} \& R_{2}\right)$ and different azimuthal numbers $\left(I_{c}=1,2,3\right)$ for two cases (left) fixed value of pressure $(P=10 \mathrm{k} \cdot \mathrm{bar})$ and different values of temperatures $(T=100,200,300,400 \mathrm{~K})$, (right) fixed value of temperature $(T=4 \mathrm{~K})$ and different values of pressures $(P=10,20,30,40 \mathrm{~K} \cdot \mathrm{bar})$. 


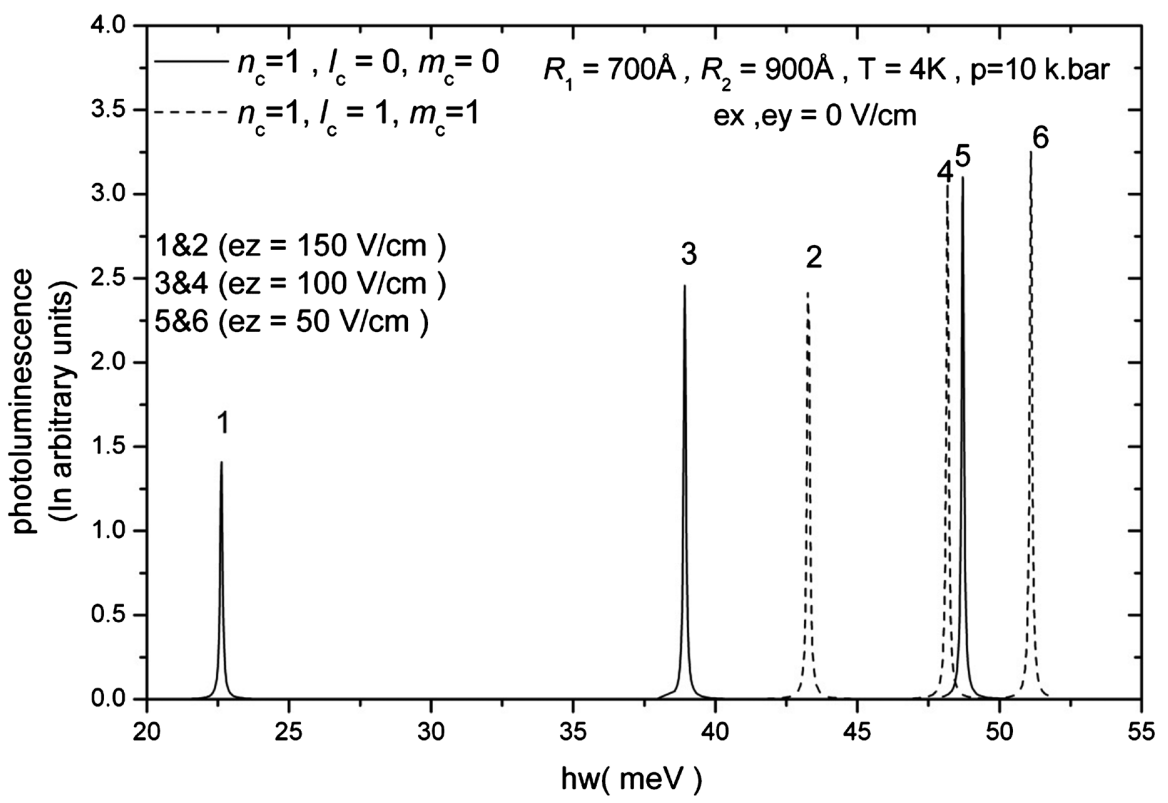

Figure 2. Represent the photoluminescence (in arbitrary units) versus the frequency of incident hole to electron transition $1 \mathrm{~h} \rightarrow 1 \mathrm{e}$ (in $\mathrm{meV}$ ) at different value of electric field component $\left(e_{x}=0, e_{y}=0, e_{z}=50,100,150 \mathrm{~V} / \mathrm{cm}\right)$ and fixed values of inner radius $\left(R_{1}=\right.$ $700 \AA)$, outer radius $\left(R_{2}=900 \AA\right)$, pressure $(P=10 \mathrm{k} \cdot \mathrm{bar})$, temperature $(T=4 \mathrm{~K})$ and different azimuthal numbers $\left(I_{c}=1,2,3\right)$.

frequency of incident hole to electron transition $1 \mathrm{~h} \rightarrow 1 \mathrm{e}$ (in $\mathrm{meV}$ ) at different value of electric field component $\left(e_{x}=0, e_{y}=0, e_{z}=50,100,150 \mathrm{~V} / \mathrm{cm}\right)$ and fixed values of inner radius $\left(R_{1}=300 \AA\right)$, outer radius $\left(R_{2}=900 \AA\right)$, pressure $(P=10$ k.bar), temperature $(T=4 \mathrm{~K})$ and different azimuthal numbers $\left(I_{c}=1,2,3\right)$. From the figure we see that with increasing the electric field component $e_{z}$ there was a red-shift in photoluminescence peak energy (wave length increase) which can be explained according to [12] [13] were the ground states energy go down with increasing the electric field due to lowering of the bottom of the well. In addition to that we see that the intensity of EL spectra decrease with increasing the applied electric field which may be attributed to the decrease in energy of the electron which proportional to the second power degree of the electric field [13] [16] [17]. Also we see that the quantum numbers $\left(n_{\mathcal{c}} l_{\mathcal{c}}, m_{c}\right)$ have a significant effects on photoluminescence peak energy at fixed value of applied electric field $\left(e_{z}\right)$ which can be explained as Ref [10] where the authors concluded that to have more than one excitons by dots the probability must be considered, and must include other factors as relaxation time, radiative recombination rate, scattering, and others.

\section{Conclusion}

The photoluminescence spectra of SLQDs were investigated theoretically under the presence of external parameters (pressure, temperature, electric field), a red-shift in photoluminescence peak was observed as a result of existing both temperature and the applied electric field while a blue-shift in photolumines- 
cence peak was observed in presence of applied hydrostatic pressure. In addition to that the quantum azimuthal number has a significant effect on photoluminescence peak energy. The study reveals the importance of such parameters in photoluminescence spectra as fitting parameters.

\section{Highlights}

- A red-shift in PL, EL peaks energy was observed with increasing both the temperature and the applied electric field while a blue-shift was observed in PL peak with increasing the hydrostatic pressure.

- The photoluminescence peak energy increase with increasing the quantum azimuthal number.

\section{Conflicts of Interest}

The author declares no conflicts of interest regarding the publication of this paper.

\section{References}

[1] Muraille, G., et al. (2020) Medium Effect on $\mathrm{Cd}_{3} \mathrm{P}_{2}$ Quantum Dots Photoluminescence and Addition of Pt Nanoparticles: Inner Filter Effect and Screening Phenomena. Journal of Luminescence, 217, Article ID: 116778.

https://doi.org/10.1016/j.jlumin.2019.116778

[2] Wang, C.Y., et al. (2020) Temperature Dependence of Photoluminescence Properties in InP/ZnS Core-Shell Quantum Dots. Journal of Luminescence, 225, Article ID: 117354. https://doi.org/10.1016/j.jlumin.2020.117354

[3] Juan, F.Y., et al. (2020) Photoluminescence Enhancement of Perovskite $\mathrm{CsPbBr}_{3}$ Quantum Dots by Plasmonic Au Nanorods. Chemical Physics, 530, Article ID: 110627. https://doi.org/10.1016/j.chemphys.2019.110627

[4] Jung, K., et al. (2020) Highly Luminescent and Stable $\mathrm{CH}_{3} \mathrm{NH}_{3} \mathrm{PbBr}_{3}$ Quantum Dots with 91.7\% Photoluminescence Quantum Yield: Role of Guanidinium Bromide Dopants. Journal of Alloys and Compounds, 832, Article ID: 154990. https://doi.org/10.1016/j.jallcom.2020.154990

[5] Park, Y.J., et al. (2020) Solution Processed Graphene Quantum Dots Decorated ZnO Nanoflowers for Mediating Photoluminescence. Applied Surface Science, 510, Article ID: 145407. https://doi.org/10.1016/j.apsusc.2020.145407

[6] Kuruoğlu, N.A., et al. (2017) Investigation of Carrier Conduction Mechanism over InAs/InP Quantum Dashes and InAs/GaAs Quantum Dots Based p-i-n Laser Heterostructures. Journal of Materials Science and Chemical Engineering, 5, 1-9. https://doi.org/10.4236/msce.2017.59001

[7] Li, C.H., et al. (2019) Ultrasonic Synthesis of $\mathrm{Mn}$-Doped $\mathrm{CsPbCl}_{3}$ Quantum Dots (QDs) with Enhanced Photoluminescence. Optical Materials, 94, 41-46. https://doi.org/10.1016/j.optmat.2019.04.053

[8] Mai, X.-D., et al. (2020) Scalable Synthesis of Highly Photoluminescence Carbon Quantum Dots. Materials Letters, 268, Article ID: 127595. https://doi.org/10.1016/j.matlet.2020.127595

[9] De Angelis, R., et al. (2013) Chemical Sensitivity of Luminescent Epitaxial Surface InP Quantum Dots. Journal of Sensor Technology, 3, 1-5. https://doi.org/10.4236/jst.2013.31001 
[10] Martini, S., et al. (2011) Radiative Recombination Mechanisms of Large InAs/GaAs Quantum Dots. World Journal of Condensed Matter Physics, 1, 161-166.

[11] Zuhair, M., et al. (2008) Interband Transition in Narrow Gap InSb Spherical Layer Quantum Dot in the Presence of Electric Field. Journal of Physics. Conference Series, 129, Article ID: 012017. https://doi.org/10.1088/1742-6596/129/1/012017

[12] Marwan, Z. (2008) Interband Transitions in a Narrow-Band Spherical InSb Quantum Layer in the Presence of an Electric Field. Journal of Contemporary Physics (Armenian Academy of Sciences), 43, 287-292. https://doi.org/10.3103/S1068337208060066

[13] Zuhair, M. (2012) Hydrostatic Pressure and Electric-Field Effects on the Electronic and Optical Properties of InAs Spherical Layer Quantum Dot. Physica E, 46, 232-235. https://doi.org/10.1016/j.physe.2012.09.017

[14] Zoheir, M., et al. (2008) Electronic States and the Stark Shift in Narrow Band InSb Quantum Spherical Layer. Physica E, 40, 2945-2949.

https://doi.org/10.1016/j.physe.2008.02.013

[15] Mkrtchyan, M.A., et al. (2020) Optical Parameters of Coupled Vertical Cylindrical Quantum Dots with Double Modified Pöschl-Teller Potential in Terahertz Range. Proceeding of SPIE, 11345, 113452B-1. https://doi.org/10.1117/12.2555456

[16] Golovynskyi, S., et al. (2020) Near-Infrared Lateral Photoresponse in InGaAs/GaAs Quantum Dots. Semiconductor Science and Technology, 35, Article ID: 055029. https://doi.org/10.1088/1361-6641/ab7774

[17] Xia, C.X., et al. (2008) Hydrostatic Pressure Effects on Exciton States in InAs/GaAs Quantum Dots. Superlattices and Microstructures, 43, 285-291. https://doi.org/10.1016/j.spmi.2007.12.001 